\title{
SMALL ANGLE NEUTRON SCATTERING (SANS) STUDIES OF DIFFUSION IN BULK POLYSTYRENE NEAR THE GLASS TRANSITION TEMPERATURE $\left(T_{g}\right)^{*}$ )
}

\author{
James E, Anderson (1)* and J,H. Jou (2) \\ Department of Nuclear Engineering, University of Michigan, \\ Ann Arbor, Michigan 48109 USA
}

\begin{abstract}
Ultraslow diffusion in bulk polymers has been measured by SANS. The experiment begins by measuring scattering from heterogeneous specimens containing domains of protonated-and deuterated-polymers at temperatures far below $\mathrm{T}_{\mathrm{g}}$. The samples are subsequently held [annealed] above $\mathrm{T}_{\mathrm{g}}$ for a known time-interval, then cooled below $\mathrm{T}_{\mathrm{g}}$ where SANS is measured again. Scattering changes, from before to aftêr annealing, are analysed to obtain diffusion coefficients. The recent Summerfield Ullman procedure is used to deconvolute portions of the scattering curve that decrease and increase with annealing time. Because of SANS sensitivity to small distances, the method yields $\mathrm{D} \approx 10^{-18}$ to $10^{-15}$ $\mathrm{cm}^{2} / \mathrm{s}$ after annealing times of $1-24 \mathrm{~h}$. Data analysis is complicated by "smearing effects" which produce apparent Q-dependent diffusion coefficients. Representative experimental results on polystyrene at $108^{\circ}-130{ }^{\circ} \mathrm{C}$ are discussed.
\end{abstract}

\section{INTRODUCTION}

Scattering/diffusion experiments have been performed on binary metal systems for many years (Ref.1). The basic technique consists of forming a heterogeneous, non-equilibrium specimen at low temperature where diffusion is effectively zero. X-ray/neutron scattering from this specimen is measured. The sample is then annealed, for a known period of time, at a temperature where interdiffusion modifies the original segregated domains. Finally, the sample is cooled and scattering is measured again. Diffusion coefficients are computed from scattering changes with annealing time. Scattering/diffusion methods provide independent measures of diffusion at each scattering angle; for this reason they are uniquely able to explore the presence or absence of Q-dependent diffusion.

Diffusion effects on scattering from binary metals is indicated schematically below. Note that scattering effectively decays to zero for a fully-annealed sample, where the two components are uniformly mixed.

*) This research forms part of the Ph.D. thesis of J.H. Jou, submitted to the University of Michigan Macromolecular Science Program [1986]. The work was sponsored by NSF Grant DMR 8217460 as an Industry/University Cooperative Research Activity.

(1) Permanent Address: Research Staff, Ford Motor Company, Dearborn, Michigan 48121 USA

(2) Permanent Address; IBM Corporation, 650 Harry Road, San Jose, California 95120 USA 


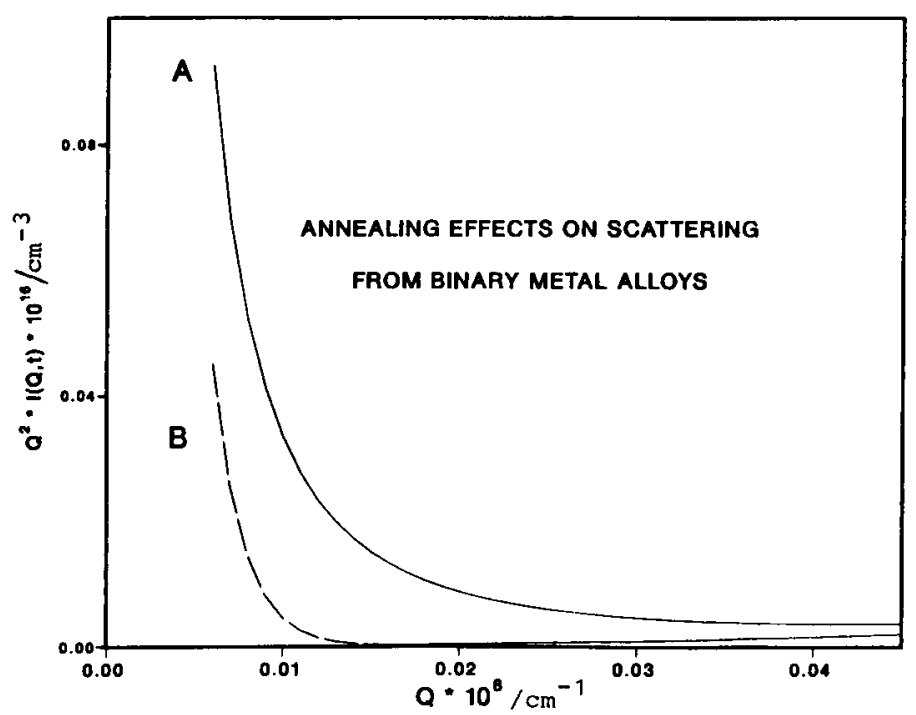

Fig. 1. Computer-simulation of SANS diffusion experiment for a binary metal system. Curve (A) represents SANS from initial specimen, plotted as $Q^{2} I(Q, 0)$ versus $Q$. Curve (B) represents a specimen annealed for intermediate time. Fully annealed specimens exhtbit no appreciable scattering over this Q-range.

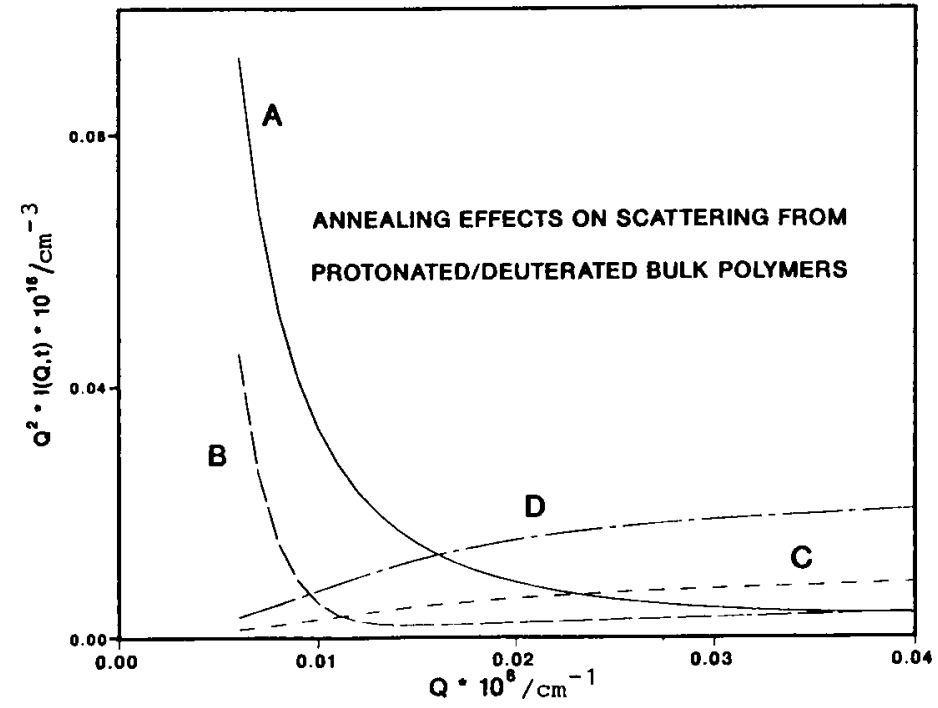

Fig. 2. Computer-simulation of SANS diffusion experiment for a polymer system. Curve (A) represents SANS from initial specimen, plotted as $Q^{2} \mathrm{I}(\mathrm{Q}, 0)$ versus $Q$. Curves (B) and (C) represent specimens annealed for intermediate times; Curve (D), a fully annealed specimen. 
Analogous diffusion experiments on protonated/deuterated polymers are complicated by the finite scattering of homogeneous samples. This difference results from the macromolecular and monomeric dimensions of polymers and metals, respectively. As a consequence of this, polymer SANS experiments typically exhibit [a] a low-Q portion that decreases with annealing time, and [b] a higher-Q portion that increases with annealing time.

Summerfield and U1lman (ref, 2) recently developed a method for deconvoluting decreasing and increasing portions of scattering/annealing curves. They showed that $I(Q, t)$, the SANS after annealing time $t$, can be expressed

$$
I(Q, t)=I(Q, 0) \exp \left(-2 D Q^{2} t\right)+a(t) I(Q, \infty)
$$

where $I(Q, 0)$ and $I(Q, \infty)$ are the scattering intensities from the initial and fully-annealed samples. The factor $a(t)$, which increases to 1 as $t \rightarrow \infty$, involves the integral of a space-correlation function. Decreasing and increasing parts of the scattering curve are not independent: They are related by the invariance of the integrated scattering intensity over all angles. One can obtain diffusion coefficlents from either part of the scattering curve. In practice, it seems easier to use the decaying contribution since it is independent of the initial distribution of heterogeneities; $1 . e$, independent of $I(Q, 0)$. On the other hand, analysing the increasing portion of the scattering curve requires information about $I(Q, 0)$ over all scattering angles, including angles below the experimental limit of SAXS and SANS instrumentation.

\section{EXPERIMENTAL}

Our experiments used 50:50 [wt 8 ] protonated/deuterated polystyrene samples having matched molecular weights of $68000,110000,240000$ and 600000 . The heterogeneous specimens, formed by freeze-drying of latex suspensions, resembled a collection of spheres with a $900 \mathrm{~nm}$ average diameter. A detafled description of sample preparation appears elsewhere (ref. 3 ).

SANS measurements were carried out at the HFIR facility at Oak Ridge National Laboratory. The majority of the diffusion measurements was done at a sample-to-detector flight path of $16.5 \mathrm{~m}$, a neutron wavelength of $4.75 \mathrm{x}$ $10^{-8} \mathrm{~cm}$ and source/sample slits of 2.0 and $1.0 \mathrm{~cm}$, respectively. Some preliminary experiments were performed at $6.89 \mathrm{~m}$.

\section{RESULTS}

The Summerfield-Ullman analysis generated $F(Q, t)-F(Q, 0)$ exp $\left(-2 D Q^{2} t\right)$ from raw experimental data. Plots of $\ln \left(F(Q, t) / F(Q, 0)\right.$ ) versus $\left(Q^{2} t\right)$ were used to obtain the diffusion coefficients. Note that $t$ and $Q^{2}$ are conjugate variables in the case of simple diffusion. In order to optimize saNS beam-time, most of our experiments measured $F(Q, t)$ at several $Q$-values and two annealing times. The intrinsic scatter from a small number of points produced a $50 \%$ uncertainty in diffusion coefficients (Table I).

More extensive measurements were performed on a specimen of $68000 \mathrm{MW}$ polystrene at $130{ }^{\circ} \mathrm{C}$ : eleven different annealing times were used. We derived diffusion coefficients as

$$
D_{t}=-0.5\left\{d \ln [F(Q, t) / F(Q, 0)] / d\left(Q^{2}\right)\right\}_{t}
$$


from data at fixed annealing time $t$ and variable $Q$, and as

$$
D_{Q}=-0.5(d \ln [F(Q, t) / F(Q, 0)] / d t]_{Q}
$$

from data at constant $Q$ and variable $t$. These values should be identical if $D$ were independent of $Q$ and $t$. As shown below, we encountered systematic departures from predictions of simple diffusion theory indicating a $2 \mathrm{X}$ variation of $D$ over the range $4 \times 10^{5}<Q<8.0 \times 10^{5} \mathrm{~cm}^{-1}$.

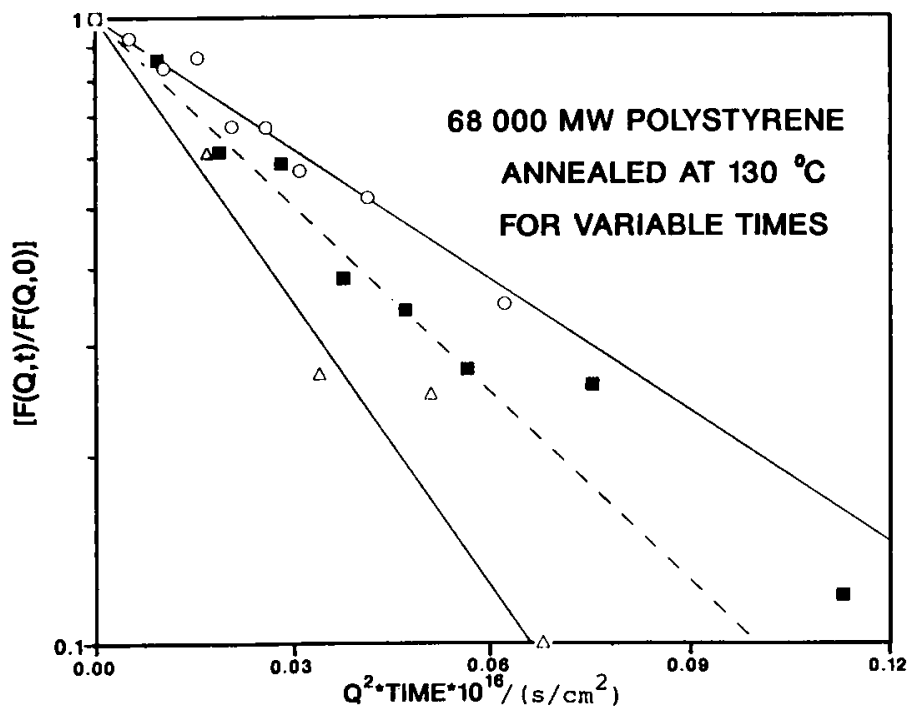

Fig. 3. Decay of experimental SANS with annealing time. Data, on $68000 \mathrm{MW}$ polystyrene at $130^{\circ} \mathrm{C}$, were taken at fixed scattering angles and various times. Circles, $Q$ $4.15 \times 10^{5} \mathrm{~cm}^{-1}$; squares, $Q-5.60 \times 10^{5} \mathrm{~cm}^{-1}$; triangles, Q- $7.52 \times 10^{5} \mathrm{~cm}^{-1}$. 


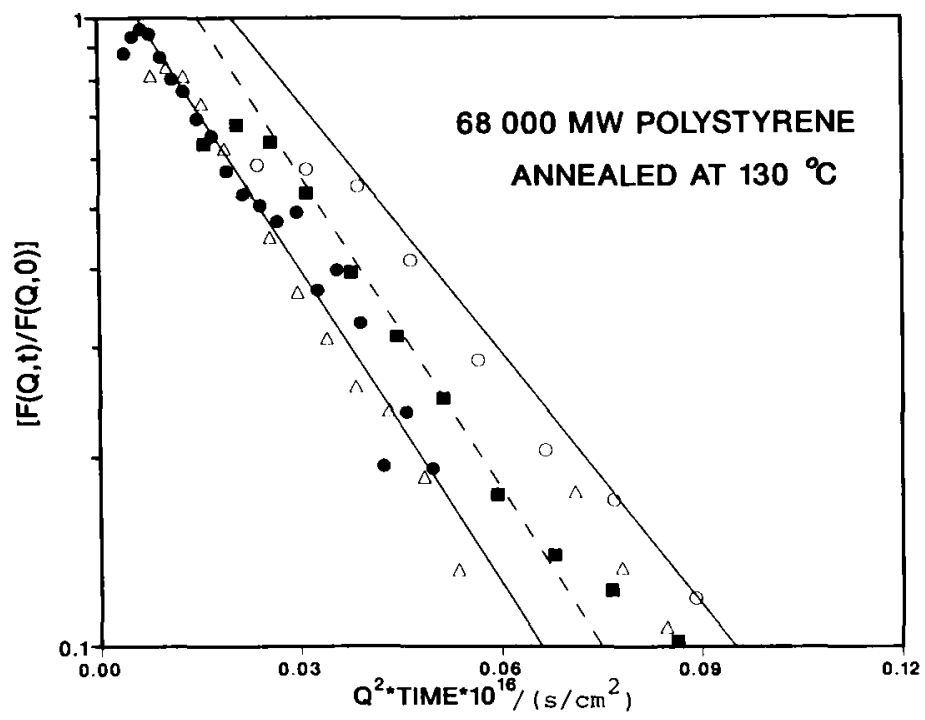

Fig. 4. Decay of experimental SANS with scattering angle $\left(Q^{2}\right)$ at fixed annealing times. Filled circles, $t=300 \mathrm{~s}$; triangles, $t=600 \mathrm{~s} ; \quad$ filled squares, $t=1200 \mathrm{~s}$; open circles, $t=1800$ $\mathrm{s}$

Experimental SANS decreases abruptly with scattering angle. At low-Q, $I(Q, 0)$ varies as $Q^{-m}$ where $m 1$ ies between 4 and 5 , whereas at higher-Q, $I(Q, 0)$ varies as $Q^{-2}$. This led us to examine possible slit-smearing effects on our data. Using instrumental parameters for the Oak Ridge facility, kindly supplied by Dr. G.D. Wignall, we calculated an instrumental broadening function of $\Delta \mathrm{Q}_{\mathrm{T}}=2.1 \times 10^{5} \mathrm{~cm}^{-1}$ (ref. 3). This was used to simulate smearing effects at the detector $\left[\mathrm{I}_{\mathrm{d}}\right]$ as:

$$
I_{d}\left(Q_{0}, t\right)=\int I_{S}(Q, t) \exp \left(-2.77\left(\left(Q-Q_{0}\right) / \Delta Q_{T}\right)^{2}\right) d\left(Q-Q_{0}\right)
$$

$I_{S}$, the sample scattering intensity, was assumed to have the form

$$
I_{S}(Q, t)=\left\{C_{1} R_{C}^{3} /\left(1+\left(Q_{c}\right)^{2}\right)^{2}\right) \exp \left(-2 D Q^{2} t\right\}
$$

where $R_{c}$ is a correlation length. The coefficient $c_{1}$ is given by

$$
\mathrm{C}_{1}=8 \pi \phi(1-\phi)\left(\mathrm{N}_{\mathrm{A}} \rho / \mathrm{M}_{\mathrm{M}}\right)^{2}\left(\mathrm{a}_{\mathrm{H}^{-}} \mathrm{a}_{\mathrm{D}}\right)^{2},
$$

where $\mathrm{N}_{A}$ is Avogadro's number, $\boldsymbol{d}$ is the volume fraction deuterated polymer, $\rho$ is the density, $M_{M}$ is the monomer $M W$, and $a_{H}$, a $a_{D}$ are the neutron crosssections for the $\mathrm{C}_{8} \mathrm{H}_{8}$ and $\mathrm{C}_{8} \mathrm{D}_{8}$ monomer units. Results of this simulation indicate that the apparent $Q$-dependence of experimental diffusion coeffi- 
cients can be attributed to smearing effects. Additional experiments are planned, using different instrumental geometries, to validate this. Note that the experimental D-values shown in Table I are not corrected for smearing effects.

\section{TABLE I}

SANS Diffusion Coefficients for 50:50

Protonated/deuterated Polystyrenes (HPS/DPS)

DPS-MW/HPS-MW ${ }^{\text {a) }}$ $\mathrm{T} /{ }^{\circ} \mathrm{C}$ $D /\left(\mathrm{cm}^{2} / \mathrm{s}\right)$

$\begin{array}{lll}68000 / 68000 & 130 & 1.85 \times 10^{-15} \\ 120 & 2.5 \times 10^{-16} \\ 108 & 7.0 \times 10^{-18} \\ 110000 / 110000 & 130 & 1.1 \times 10^{-15} \\ 120 & 1.6 \times 10^{-16} \\ 240000 / 233000 & 130 & 1.8 \times 10^{-18} \\ 697000 / 600000 & 120 & 7.5 \times 10^{-17} \\ 130 & 6.8 \times 10^{-18} \\ & 130 & 1.7 \times 10^{-17}\end{array}$

a) ${ }_{\mathrm{MW}}=$ molecular weight

\section{REFERENCES}

1. J. DuMond and J.P. Youtz, J. Appl. Phys 11, 357 (1940).

2. G.C. Summerfield and R. Ullman, Macromolecules 20, 401 (1987).

3. J.E. Anderson and J.H. Jou, Macromolecules 20, 1544 (1987). 